\title{
Vorwort der Herausgeber
}

Niemanden, der sich ernsthaft mit FGM beschäftigt, lässt dieses Problem emotionslos zurück. FGM ist ein grausames Ritual, dass beendet werden muss. Das Problem lässt sich aber nur ganzheitlich, gemeinsam und Schritt für Schritt lösen. Zunächst geht es darum, die Hintergründe und jahrhundertealten Traditionen von FGM zu verstehen. Dazu muss man bereit sein, eigene Traditionen $\mathrm{zu}$ hinterfragen und immer wieder die eigene begrenzte Perspektive zu verlassen. Das geht nur, wenn man Betroffenen zuhört und lernt, was FGM wirklich bedeutet. Aber auch was es bedeutet, sich von eigenen Traditionen lösen zu wollen. Vielen wird unbekannt sein, dass in westlichen Ländern, auch in Deutschland, die weibliche Genitalbeschneidung Ende des 19. bis weit in das 20. Jahrhundert hinein zur Behandlung von „Frauenleiden“ wie Hysterie, Nervosität und Nymphomanie aber auch Epilepsie vorgenommen wurde. Daher kommen in diesem Buch viele Betroffene zu Wort. Ohne die Sicht der Betroffenen kann man den umfassenden Kontext von FGM nicht erfassen. Hierin zeigt sich welche lebenslangen Folgen FGM für die Mädchen und Frauen haben kann. Dann ist diese nachhaltige Beschäftigung mit FGM ein Prozess, der auch uns „Helfende“ verändern wird. Sie wird unsere Perspektive verändern.

Dieses Buch soll allen die sich mit FGM beschäftigen, dabei Hilfestellungen geben. Wir haben versucht, alle Aspekte von FGM zu beleuchten. Wir wollen darstellen, dass jeder etwas tun kann, egal in welchem psychosozialen oder medizinischen Umfeld er sich bewegt. Wir dürfen nicht akzeptieren, dass Mädchen so etwas angetan wird.

Wir bedanken uns bei allen Betroffenen, die ihr Schicksal offenbart haben und so das furchtbare Tabu brechen, darüber nicht zu sprechen. Wir danken allen Autoren, die ihr Wissen und ihre Erfahrung in diesem Buch dargestellt haben. Wir danken allen Leserinnen und Lesern, denen das Buch neue Aspekte eröffnen wird.

Nur gemeinsam können wir dieses grausame Ritual beenden.

Uwe von Fritschen, Cornelia Strunz, Roland Scherer 
